\title{
Fast Response Multilevel Scheduling Scheme for Wireless Sensor Networks
}

\author{
${ }^{1}$ Mrs. Vaishali S. Nikam, ${ }^{2}$ Prof. Shiv Om Tiwari \\ $2^{\text {nd }}$ Year M.E. (E \& TC) Siddhartha Collage of Engineering Talegaon, Pune. Maharashtra \\ (Asst. Professor) Siddhartha Collage of Engineering Malegaon, Pune. Maharashtra ${ }^{2}$
}

\begin{abstract}
Wireless sensor networks (WSNs) have a wide variety of military and civil applications. The sensor nodes are powered by batteries with limited energy. Hostile or hazardous environments where the sensor nodes are deployed or the sheer number of the sensors prevents replacement or recharge of the batteries. Therefore in WSNs, scheduling of different types of packets is of vital importance to reduce sensors' energy consumptions and end-to-end data transmission delays. Most of the existing packet-scheduling mechanisms of WSN use First Come First Served (FCFS), non-preemptive priority and preemptive priority scheduling algorithms. These algorithms incur a high processing overhead and long end-to-end data transmission delay due to the FCFS concept, starvation of high priority real-time data packets due to the transmission of a large data packet in nonpreemptive priority scheduling, starvation of non-real-time data packets due to the probable continuous arrival of real-time data in preemptive priority scheduling, and improper allocation of data packets to queues in multilevel queue scheduling algorithms. Moreover, these algorithms are not dynamic to the changing requirements of WSN applications since their scheduling policies are predetermined. In this paper, we propose a Dynamic Multilevel Priority (DMP) packet scheduling scheme. In the proposed scheme, each node, except those at the last level of the virtual hierarchy in the zone-based topology of WSN, has three levels of priority queues. Real-time packets are placed into the highest-priority queue and can preempt data packets in other queues. Non-real-time packets are placed into two other queues based on a certain threshold of their estimated processing time. Leaf nodes have two queues for real-time and non-real-time data packets since they do not receive data from other nodes and thus, reduce end-to-end delay.
\end{abstract}

Keywords: Data waiting time, FCFS, Wireless sensor network, non-preemptive priority scheduling, packet scheduling, preemptive priority scheduling, real-time, non-real-time.

\section{Introduction}

Wireless sensor networks usually contain thousands of sensors, which are randomly and densely deployed. Each sensor has a light weight and a low cost with three technologies of sensing, on-board processing and transmission. Sensor nodes have limited battery power which leads to limited coverage and communication range.

Most of the applications in wireless sensor networks involve primarily data aggregation in which sensor node periodically produced data and transmitting to the sink node through the aggregated node where continuous queries are posed and processed. But data aggregations in WSN have two main issues: First, save energy in battery powered sensor and second, fast and efficient query response are essential to network performance and maintenance. In sensor node, both sensor element and processing element consume constant and low power. Energy used by the transceiver is variable and very high in comparison to sensing and processing energy. The power consumed in the transmission depends upon the network topology, MAC layer protocol, routing algorithms, data fusion and cache memory in sensor node.

Among many network design issues, such as routing protocols and data aggregation, that reduce sensor energy consumption and data transmission delay, packet scheduling (interchangeably use as task scheduling) at sensor nodes is highly important since it ensures delivery of different types of data packets based on their priority and fairness with a minimum latency. For instance, data sensed for real-time applications have higher priority than data sensed for non-real- time applications. Though extensive research for scheduling the sleepwake times of sensor nodes has been conducted, only a few studies exist in the literature on the packet scheduling of sensor nodes that schedule the processing of data packets available at a sensor node and also reduces energy consumptions. Indeed, most existing Wireless Sensor Network (WSN) operating systems use First Come First Serve (FCFS) schedulers that process data packets in the order of their arrival time and, thus, require a lot of time to be delivered to a relevant base station (BS). However, to be meaningful, sensed data have to reach the BS within a specific time period or before the expiration of a deadline. Additionally, real-time emergency data should be delivered to BS with the shortest possible end-to-end delay. Hence, intermediate nodes require changing the delivery order of data packets in their ready queue based on their importance (e.g., real or non-real time) and delivery deadline. Further- more, most existing packet scheduling algorithms of WSN 
are neither dynamic nor suitable for large scale applications since these schedulers are predetermined and static, and cannot be changed in response to a change in the application requirements or environments . For example, in many real- time applications, a real-time priority scheduler is statically used and cannot be changed during the operation of WSN applications.

In this paper, a Dynamic Multilevel Priority (DMP) packet scheduling scheme for WSNs in which sensor nodes are virtually organized into a hierarchical structure. Nodes that have the same hop distance from the BS are considered to be located at the same hierarchical level. Data packets sensed by nodes at different levels are processed using a TDMA scheme. For instance, nodes that are located at the lowest level and one level upper to the lowest level can be allocated timeslots 1 and 2, respectively. Each node maintains three levels of priority queues. This is because we classify data packets as (i) real-time (priority 1), (ii) non-real-time remote data packet that are received from lower level nodes (priority 2), and (iii) non-real-time local data packets that are sensed at the node itself (priority 3). Non-real-time data traffic with the same priority are processed using the shortest job first (SJF) scheduler scheme since it is very efficient in terms of average task waiting time .

\section{Literature Overview}

Many scheduling algorithms are proposed for extending the life time of wireless sensor network. Hossein Momeni [21] proposed a new approach to task allocation in wireless sensor actor network which guarantee that the task complete their activities before their deadline expires. Fadi Tirkawi[22] proposed scheduling in nodes dependent on their depth from base station so that these nodes can have better chance to participate in sensing. Priority based low power task scheduling based on battery model \& task model was proposed by Xiang $\mathrm{Yu}$ [23] to reduce energy consumption of the tasks.

\section{Related Works}

Here present existing packet or task scheduling schemes by classifying them based on several factors as is illustrated in Fig1.

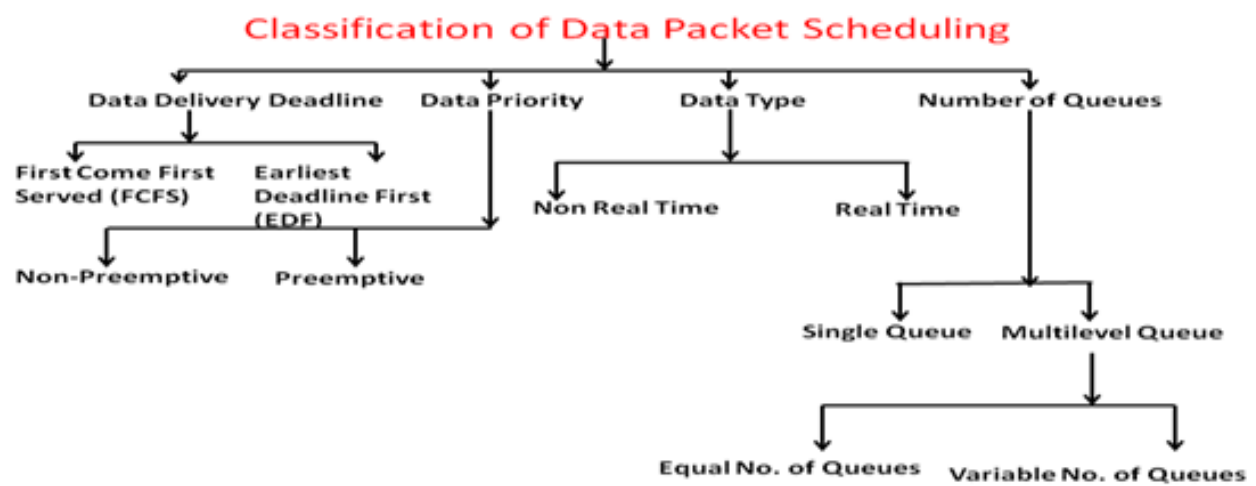

Figure 1 Classification of packet scheduling schemes.

\section{III.I Factor: Deadline}

Packet scheduling schemes can be classified based on the deadline of arrival of data packets to the base station (BS), which are as follows. First Come First Served (FCFS): Most existing WSN applications use First Come First Served (FCFS) schedulers that process data in the order of their arrival times at the ready queue. In FCFS, data that arrive late at the intermediate nodes of the network from the distant leaf nodes require a lot of time to be delivered to base station (BS) but data from nearby neighboring nodes take less time to be processed at the intermediate nodes. In FCFS, many data packets arrive late and thus, experience long waiting times.

Earliest Deadline First (EDF): Whenever a number of data packets are available at the ready queue and each packet has a deadline within which it should be sent to BS, the data packet which has the earliest deadline is sent first. This algorithm is considered to be efficient in terms of average packet waiting time and end-to-end delay. The research work done by Lu C. et al. [28] proposes a real-time communication architecture for largescale sensor networks, whereby they use a priority-based scheduler. Data, that have travelled the longest distance from the source node to BS and have the shortest deadline, are prioritized. If the deadline of a particular task expires, the relevant data packets are dropped at an intermediate node. Though this approach reduces network traffic and data processing overhead, it is not efficient since it consumes resources such as memory and computation power and increases processing delay. The performance of the scheme can be improved by incorporating FCFS. Mizanian et al. [29] proposed RACE, a packet- scheduling policy and routing algorithm for real-time large- scale sensor networks that uses a loop-free Bellman-Fordalgorithm to find paths with the minimum traffic load and delay between source and destination. RACE uses the Earliest Deadline First (EDF) 
scheduling concept to send packets with earliest deadline. It also uses a prioritized MAC protocol that modifies the initial wait time after the channel becomes idle and the back-off window increases the function of the IEEE 802.11 standard. Priority queues actively drop packets whose deadlines have expired to avoid wasting network resources. However, local prioritization at each individual node in RACE is not sufficient because packets from different senders can compete against each other for a shared radio communication channel.

\section{III.II Factor: Priority}

Packet scheduling schemes can be classified based on the priority of data packets that are sensed at different sensor nodes.

Non-preemptive: In non-preemptive priority packet scheduling, when a packet t1 starts execution, task $\mathrm{t} 1$ carries on even if a higher priority packet $\mathrm{t} 2$ than the currently running packet $\mathrm{t} 1$ arrives at the ready queue. Thus $\mathrm{t} 2$ has to wait in the ready queue until the execution of $\mathrm{t} 1$ is complete.

Preemptive: In preemptive priority packet scheduling, higher priority packets are processed first and can preempt lower priority packets by saving the context of lower priority packets if they are already running. The widely used operative system of WSN and classify them as either cooperative or preemptive. Cooperative scheduling schemes can be based on a dynamic priority scheduling mechanism, such as EDF and Adaptive Double Ring Scheduling (ADRS), that uses two queues with different priorities. The scheduler dynamically switches between the two queues based on the deadline of newly arrived packets. If the deadlines of two packets are different, the shorter deadline packet would be placed into the higher-priority queue and the longer deadline packet would be placed into the lower-priority one. Cooperative schedulers in TinyOS are suitable for applications with limited system resources and with no hard real-time requirements. On the other hand, preemptive scheduling can be based on the Emergency Task First Rate Monotonic (EF-RM) scheme. EF-RM is an extension to Rate Monotonic (RM), a static priority scheduling, whereby the shortest-deadline job has the highest priority. EF-RM divides WSN tasks into Period Tasks, (PT) whose priorities are decided by a RM algorithm, and non- period tasks, which have higher priority than PTs and can interrupt, whenever required, a running PT.

\section{III.III Factor: Packet Type}

Packet scheduling schemes can be classified based on the types of data packets, which are as follows. Real-time packet scheduling: Packets at sensor nodes should be scheduled based on their types and priorities. Real-time data packets are considered as the highest priority packets among all data packets in the ready queue. Hence, they are processed with the highest priority and delivered to the BS with a minimum possible end-to-end delay.

\section{Non-real-time packet scheduling:}

Non-real time packets have lower priority than real-time tasks. They are hence delivered to BS either using first come first serve or shortest job first basis when no real-time packet exists at the ready queue of a sensor node. These packets can be intuitively preempted by real-time packets.

Though packet scheduling mechanisms of TinyOS are simple and are used extensively in sensor nodes, they cannot be applied to all applications: due to the long execution time of certain data packets, real-time packets might be placed into starvation. Moreover, the data queue can be filled up very quickly if local data packets are more frequent that causes the discard of real-time packets from other nodes. To eliminate these drawbacks, Zhao Y. proposed an improved priority-based soft real-time packet scheduling algorithm. Schedulers traverse the waiting queue for the data packets and choose the smallest packet ID as the highest priority to execute. Each packet is assigned an Execute Counter, EXECUTE MAX TIME, i.e., the largest initial task execution time. The management component compares the current packet ID with the previous packet ID. If it is the same, the system executes it and decrements the counting variable. Otherwise, if the counting variable is null, the management component terminates this packet and other packets get the opportunity to be executed. However, packet priorities are decided during the compilation phase, which cannot be changed during the execution time. If high priority packets are always in execution, the low priority packets cannot be implemented. If low-priority packets occupy the resources for a long time, the subsequent high-priority packets cannot get response in time.

\section{III.IV Factor: Number of Queue}

Packet scheduling schemes can also be classified based on the number of levels in the ready queue of a sensor node. These are as follows. 
Single Queue: Each sensor node has a single ready queue. All types of data packets enter the ready queue and are scheduled based on different criteria: type, priority, size, etc. Single queue scheduling has a high starvation rate.

Multi-level Queue: Each node has two or more queues. Data packets are placed into the different queues according to their priorities and types. Thus, scheduling has two phases: (i) allocating tasks among different queues, (ii) scheduling packets in each queue. The number of queues at a node depends on the level of the node in the network. For instance, a node at the lowest level or a leaf node has a minimum number of queues whilst a node at the upper levels has more queues to reduce end-to-end data transmission delay and balance network energy consumptions. Figure 4 illustrates the main concept behind multi-level queue scheduling algorithms.

To eliminate problems in proposed a multilevel queue scheduler scheme that uses a different number of queues according to the location of sensor nodes in the network. This approach uses two kinds of scheduling: simple priority-based and multi-FIFO queue-based. In the former, data enter the ready queue according to priority but this scheduling also has a high starvation rate. The multi-FIFO queue is divided into a maximum of three queues, depending on the location of the node in the network. If the lowest level is, nodes that are located at level have only one queue but there are two queues for nodes at level . Each queue has its priority set to high, mid, or low. When a node receives a packet, the node decides the packet's priority according to the hop count of the packet and accordingly sends it to the relevant queue. The work done by Karimi E. and Akbari B. also proposes a priority queue scheduling algorithm for WMSN. In this scheduling scheme, buffer space of intermediate nodes is divided into four queues to hold three different types of video frames and one regular data frames. Data in the first three queues have the highest priority and are scheduled in round- robin fashion. Data in the fourth queue is transmitted when the first three queues are empty. However, these scheduling schemes do not consider variable number of queues based on the position of sensor nodes to reduce the overall end-to-end delay.

\section{Preliminaries}

In this section, present general assumptions and define some terminologies that are used in designing the Dynamic Multilevel Priority (DMP) packet scheduling scheme.

\section{IV.I. Assumptions}

We make the following assumptions to design and implement DMP packet scheduling scheme.

- Data traffic comprises only real-time and non-real-time data, e.g., real-time health data sensed by body sensors and non-real-time temperature data.

- All data packets (real-time and non-real-time) are of same size.

- Sensors are time synchronized.

- No data aggregation is performed at intermediate nodes for real-time data.

- Nodes are considered located at different levels based on the number of hop counts from BS.

- Timeslots are allocated to nodes at different levels using TDMA scheme, e.g., nodes at the lowest level, lk are

- assigned timeslot 1. Details of timeslot allocation are explained in the "Terminologies" subsection.

- The ready queue at each node has maximum three levels or sections for real-time data (pr1) non-realtime remote

- data (pr2) and non-real-time local data (pr3).

- The length of data queues is variable. For instance, the length of real-time data queue (pr1) is assumed to be

- smaller than that of non-real-time data queues (pr2 and pr3). However, the length of the non-real-time pr2 and pr3

- queues are same.

- DMP scheduling scheme uses a multichannel MAC protocol to send multiple packets simultaneously.

\section{IV.II. Terminologies}

In this section, we define the following terminologies and factors that are used in designing the DMP packet scheduling scheme.

\section{IV.II.I Routing Protocol}

For the sake of energy efficiency and balance in energy consumption among sensor nodes, weenvision using a zone-based routing protocol $[4,8]$. In a zone- based routing protocol, each zone is identified by a zone head $(\mathrm{ZH})$ and nodes follow a hierarchical structure, based on the number of hops they are distant from the base 
station (BS). For instance, nodes in zones that are one hop and two hops away from the BS are considered to be at level 1 and level 2, respectively. Each zone is also divided into a number of small squares in such a way that if a sensor node exists in square S1, it covers all neighboring squares. Thus, this protocol reduces the probability of having any sensing hole in the network even if the neighboring squares of a node do not have any sensor node.

\section{IV.II.II TDMA Scheme}

Task or packet scheduling at each nodal level is performed using a TDMA scheme with variable-length timeslots. Data are transmitted from the lowest level nodes to BS through the nodes of intermediate levels. Thus, nodes at the intermediate and upper levels have more tasks and processing requirements compared to lowerlevel nodes. Considering this observation, the length of timeslots at the upper-level nodes is set to a higher value compared with the timeslot length of lower-level nodes. On the other hand, real-time and time- critical emergency

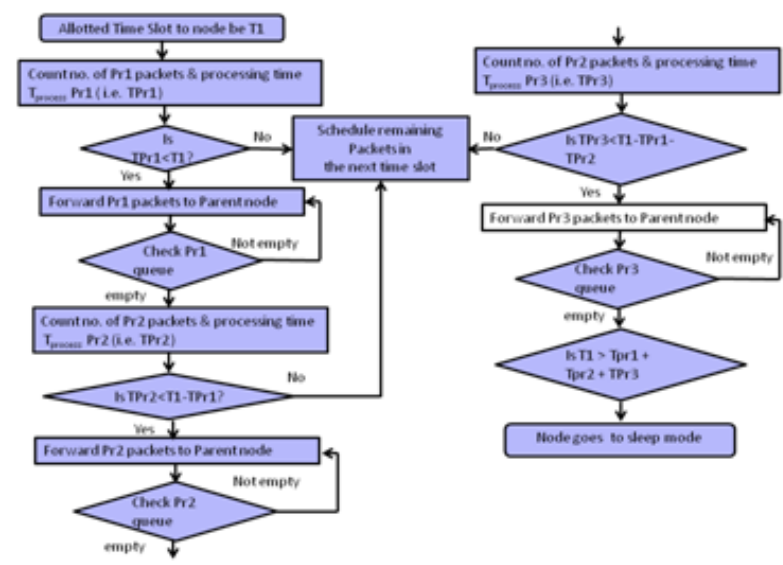

Applications should stop intermediate nodes from aggregating data since they should be delivered to end users with a minimum possible delay. Hence, for real-time data, the duration of timeslots at different levels is almost equal and short.

\section{IV.II.III Fairness}

This metric ensures that tasks of different priorities get carried out with a minimum waiting time at the ready queue based on the priority of tasks. For instance, if any lower- priority task waits for a long period of time for the continuous arrival of higher-priority tasks, fairness defines a constraint that allows the lowerpriority tasks to get processed after a certain waiting time. Priority: As discussed earlier, real-time and emergency data should have the highest priority. The priority of non-real-time data packets is assigned based on the sensed location (i.e., remote or local) and the size of the data. The data packets that are received by node $\mathrm{x}$ from the lower level nodes are given higher priority than the data packets sensed at the node $\mathrm{x}$ itself. However, if it is observed that the lower priority non-real- time local data cannot be transmitted due to the continuous arrival of higher priority non-real-time remote data, they are preempted to allow low-priority data packets to be processed after a certain waiting period. Nevertheless, these tasks can be preempted by real-time emergency tasks. In case of two same priority data packets the smaller sized data packets are given the higher priority.

\section{Proposed Dmp Packet Scheduling Scheme}

As discussed earlier, in non-preemptive packet scheduling schemes (interchangeably use as task scheduling in this paper), real-time data packets have to wait for completing the transmissions of other non-realtime data packets. On the other hand, in preemptive priority scheduling, lower-priority data packets can be placed into starvation for continuous arrival of higher-priority data. In the multilevel queue scheduling algorithm [5], each node at the lowest level has a single task queue considering that it has only local data to process.

However, local data can also be real-time or non-real time and should be thus processed according to their priorities. Otherwise, emergency real-time data traffic may experience long queuing delays till they could be processed. Thus, we pro- pose a Dynamic Multilevel Priority (DMP) packet scheduling scheme that ensures a tradeoff between priority and fairness. 


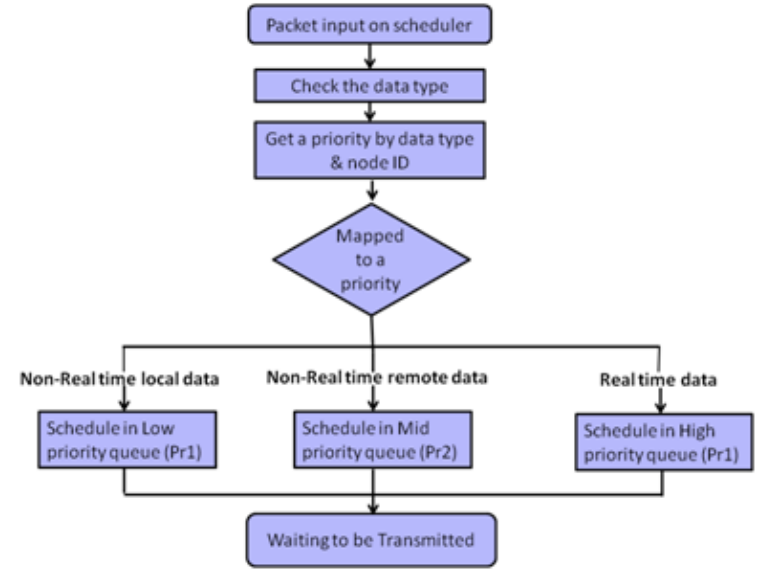

\section{V.I Working Principle[1]}

Scheduling data packets among several queues of a sensor node is presented in Figure 2. Data packets that are sensed at a node are scheduled among a number of levels in the ready queue. Then, a number of data packets in each level of the ready queue are scheduled.

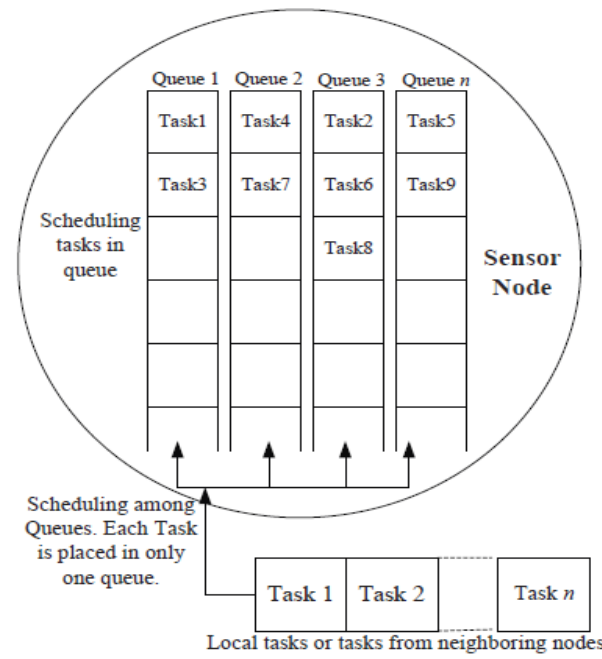

Fig. 2.Scheduling data among multiple queues.

For instance, Fig. 2 demonstrates that the data packet, Data1 is scheduled to be placed in the first level, Queue1. Then, Data1 and Data3 of Queue1 are scheduled to be transmitted based of different criteria. The general working principle of the proposed DMP scheduling scheme is illustrated in Fig3.

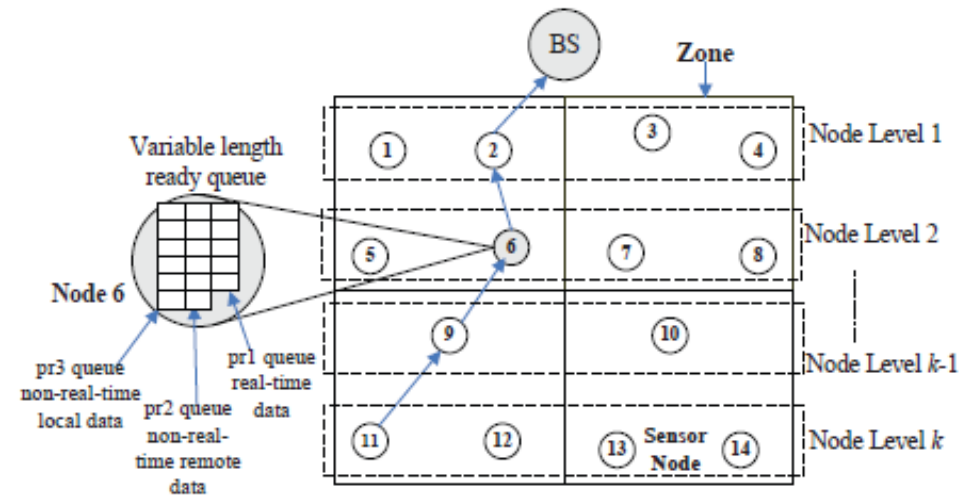

Figure 3. Proposed Dynamic Multilevel Priority Packet Scheduling Scheme 
The proposed scheduling scheme assumes that nodes are virtually organized following a hierarchical structure. Nodes that are at the same hop distance from the base station (BS) are considered to be located at the same level. Data packets of nodes at different levels are processed using the Time-Division Multiplexing Access (TDMA) scheme. For instance, nodes that are located at the lowest level and the second lowest level can be allocated timeslots 1 and 2, respectively. We consider three-level of queues, that is, the maximum number of levels in the ready queue of a node is three: priority $1\left(\mathrm{pr}_{1}\right)$, priority $2\left(\mathrm{pr}_{2}\right)$, and priority $3\left(\mathrm{pr}_{3}\right)$ queues. Realtime data packets go to $\mathrm{pr} 1$, the highest priority queue, and arepr3 queue non-real-time local data $\mathrm{pr}_{2}$ queue nonreal- time remote data pr1 queue real-time data processed using FCFS. Non-real-time data packets that arrive from sensor nodes at lower levels go to $\mathrm{pr}_{2}$, the second highest priority queue. Finally, non-real time data packets that are sensed at a local node go to pr3, the lowest priority queue. The possible reasons for choosing maximum three queues are to process (i) real-time pr1 tasks with the highest priority to achieve the overall goal of WSNs, (ii) non real-time $\mathrm{pr}_{2}$ tasks to achieve the minimum average task waiting time and also to balance the end-to-end delay by giving higher priority to remote data packets, (iii) non-real-time $\mathrm{pr}_{3}$ tasks with lower priority to achieve fairness by preempting $\mathrm{pr}_{2}$ tasks if $\mathrm{pr}_{3}$ tasks wait a number of consecutive timeslots. In the proposed scheme, queue sizes differ based on the application requirements. Since preemptive priority scheduling incurs overhead due to the context storage and switching in resource constraint sensor networks, the size of the ready queue for preemptive priority schedulers is expected to be smaller than that of the preemptable priority schedulers. The idea behind this is that the highest-priority real-time/emergency tasks rarely occur. They are thus placed in the preemptive priority task queue (pr1 queue) and can preempt the currently running tasks. Since these processes are small in number, the number of preemptions will be a few. On the other hand, nonreal-time packets that arrive from the sensor nodes at lower level are placed in the preemptable priority queue $\left(\mathrm{pr}_{2}\right.$ queue). The processing of these data packets can be preempted by the highest priority real-time tasks and also after a certain time period if tasks at the lower priority $\mathrm{pr}_{3}$ queue do not get processed due to the continuous arrival of higher priority data packets. Real-time packets are usually processed in FCFS fashion. Each packet has an ID, which consists of two parts, namely level ID and node ID. When two equal priority packets arrive at the ready queue at the same time, the data packet which is generated at the lower level will have higher priority. This phenomenon reduces the end-to-end delay of the lower level tasks to reach the BS. For two tasks of the same level, the smaller task (i.e., in terms of data size) will have higher priority.

Moreover, it is expected that when a node $\mathrm{x}$ senses and receives data from lower-level nodes, it is able to process and forward most data within its allocated timeslot; hence, the probability that the ready queue at a node becomes full and drops packets is low. However, if any data remains in the ready queue of node $\mathrm{x}$ during its allocated timeslot, that data will be transmitted in the next allocated timeslot.

Timeslots at each level are not fixed. They are rather calculated based on the data sensing period, data transmission rate, and CPU speed. They are increased as the levels progress through BS. However, if there is any real-time or emergency response data at a particular level, the time required to transmit that data will be short and will not increase at the upper levels since there is no data aggregation. The remaining time of a timeslot of nodes at a particular level will be used to process data packets at other queues. Since the probability of having real-time emergency data is low, it is expected that this scenario would not degrade the system performance. Instead, it may improve the perceived Quality of Service (QoS) by delivering real-time data fast. Moreover, if any node $\mathrm{x}$ at a particular level completes its task before the expiration of its allocated timeslot, node $\mathrm{x}$ goes to sleep by turning its radio off for the sake of energy efficiency.

\section{Performance Evaluation [1]}

In this section, we analyze the performance of the proposed DMP task scheduling scheme in terms of end-toend delay of different types of traffic at the ready queues of active nodes.

In the following, we formulate the average end-to-end delay of transmitting different priority data packets to the base station (BS). Again, we interchangeably use task and data to represent the data packets that are sensed at a sensor node.

\section{VI.I. Real-time Priority 1 Queue Data:}

Let us assume that a node $\mathrm{x}$, residing at level $\mathrm{lk}$ is sensing a real-time, emergency event, e.g., fire detection. This node transmits the emergency priority 1 data to BS through $1 \mathrm{k}-1$ intermediate levels. We consider the following scenario whereby every time a real-time data packet reaches a neighboring active node, $y$ at an upper Level, a non-real time lower priority data is being processed at that node. Hence, data delivery at y is preempted to send real-time data.

Transmission time or delay that is required to place a real time data from a node into the medium is equal to datapr1/st. The propagation time, or delay to transmit data from the source to destination can be formulated as $\mathrm{d} / \mathrm{sp}$. Considering the above mentioned scenario the end-to-end delay for sending a real time data satisfies the following inequality. 
delaypr $1 \geq \operatorname{lk} \times($ datapr $1 / \mathrm{st}+\operatorname{pr} 1 \operatorname{proc}(\mathrm{t}))+\mathrm{d} / \mathrm{sp}+(\mathrm{k} \times$ toverhead $)$

Where datapr $1=$ The real-time data size, $\mathrm{st}=$ The data transmission speed, $\mathrm{d}=$ the distance from the source node to $\mathrm{BS}$, where $\mathrm{d}=\sum_{\mathrm{i}=1}^{\mathrm{lk}}(\mathrm{di}), \mathrm{sp}=$ The propagation speed over the wireless medium, $\operatorname{pr} 1 \operatorname{proc}(\mathrm{t})=$ The processing time of real-time tasks at each node, and toverhead is an overhead in terms of context switching and queuing time (including time for preemption). However, a real-time task $\mathrm{t} 1$ has to wait if there is a number, npr1, of a real-time task ahead of $\mathrm{t} 1$ at the prl queue. We assume that all real-time data have the same size. Therefore, the end-to-end delay for a real-time task $\mathrm{t} 1$ considering that $\mathrm{t} 1$ has npr 1 number of real-time tasks ahead of it,

$$
\text { delayt } 1 \geq \sum_{\mathrm{i}=1}^{\mathrm{npr} 1}(\text { delaypr1) } \mathrm{i}
$$

VI.II. Non-real time Priority 2 Queue Data: Tasks at pr2 queue can be preempted by real-time ones. Taking the scenario of Figure 3 as an example, we first consider the scenario when a real-time task is sensed at node 11 and is forwarded to BS through relay nodes 9,6 , and 2 . It should be observed that tasks are available at the pr2 queue at nodes 9, 6 and 2. Since one real-time task is available at the pr1 queue of nodes 9, 6, and 2, real-time tasks will be processed and transmitted first during the timeslot of nodes 9,6 , and 2 . The pr 2 tasks are processed in the remaining time of the timeslots. The transmission time or delay to place pr2 data from a node into the medium can be therefore computed as datapr $2 /$ st. Thus, the total end-to-end delay for a pr 2 task that can be processed in the same timeslot exceeds

$\mathrm{lk} \times($ datapr $1 / \mathrm{st}+$ datapr $2 / \mathrm{st}+\operatorname{pr} 1 \operatorname{proc}(\mathrm{t})+\operatorname{pr} 2 \operatorname{proc}(\mathrm{t}))+\mathrm{d} / \mathrm{sp}+(\mathrm{lk} \times$ toverhead $)$

VI.III. Non-real time Priority 3 Queue Data: In the best case, when no task is available at the pr1 and pr2 queues, the end to- end delay of the pr3 tasks will be almost equal to that of the pr1 queue tasks (Equation 1) although it can differ slightly based on the size of the pr3 queue task. We assume that the pr3 queue tasks are processed by preempting pr2 queue tasks if for $\alpha$ consecutive timeslots there is no task at the pr1 queue but there are tasks available at the pr 2 queue. Let tk denote the length of a timeslot of nodes at level lk. The transmission time or delay to place pr3 data from a node into the wireless medium is equal to datapr3/st. However, during the processing of the pr3 queue tasks, these tasks can be preempted by real time tasks. They are processed again after the completion of real-time tasks. Thus, the end-to-end delay for processing pr3 tasks will be exceeding $\alpha \times \mathrm{t}(\mathrm{k})+\mathrm{lk} \times($ datapr3/st + pr3proc $(\mathrm{t}))+\mathrm{d} / \mathrm{sp})+(\mathrm{lk} \times$ toverhead $)$

\section{Results}

The simulation model is implemented using Java. It is used to evaluate the performance of the proposed DMP packet scheduling scheme, comparing it against the FCFS, and Multilevel Queue scheduling schemes. The comparison is made in terms of end-to-end data transmission delay. The ready queue of each node can hold a maximum of 50 tasks. Each task has a Type ID that identifies its type. For instance, type 0 is considered to be a real-time task. Data packets are placed into the ready queue based on the processing time of the task. Moreover, each packet has a hop count number that is assigned randomly, and the packet with the highest hop count number is placed into the highest-priority queue. We run the simulation for a specific number of in the network until data from a node in each level reach BS. Simulation results are presented for both realtime data and all types of data traffic. Table I presents simulation parameters, and their respective values.

\begin{tabular}{|l|l|}
\hline Parameter & Value \\
\hline Network Size & $100 \mathrm{~m} \mathrm{X} 100 \mathrm{~m}$ \\
\hline Number of Nodes & Maximum 200 \\
\hline Number of Zones & $4-12$ \\
\hline Base station position & $55 \mathrm{~m} \mathrm{X} 101 \mathrm{~m}$ \\
\hline Transmission Energy Consumptions & $50 \mathrm{nJoule} / \mathrm{bit}$ \\
\hline Energy Consumption in free space or air & $0.01 \mathrm{nJoule} / \mathrm{bit} / \mathrm{m}^{2}$ \\
\hline Initial Node Energy & 2 Joule \\
\hline Transmission Speed & $250 \mathrm{Kbps}$ \\
\hline Propagation Speed & $198 \times 10^{6} \mathrm{~meter} / \mathrm{sec}$ \\
\hline
\end{tabular}

TableI :Simulation Parameters \& their respective values

Fig.4 illustrate the end-to-end data transmission delay of real-time tasks over a number levels. we expect that the proposed DMP scheduling scheme outperforms the existing FCFS, and Multilevel Queue scheduler. This is because the proposed scheduling scheme gives the highest priority to real-time tasks and also allows real-time data packets to preempt the processing of non-real time data packets. Thus, real-time data packets have lower data transmission delays. 


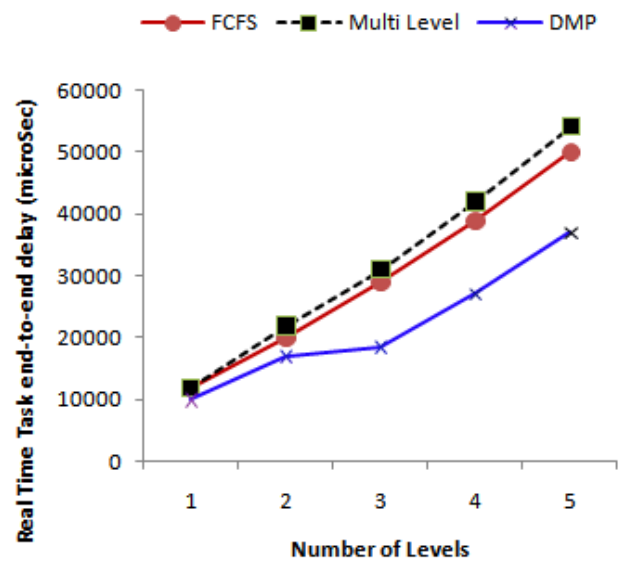

Fig.4 End-to-end delay of real time data over no. of levels

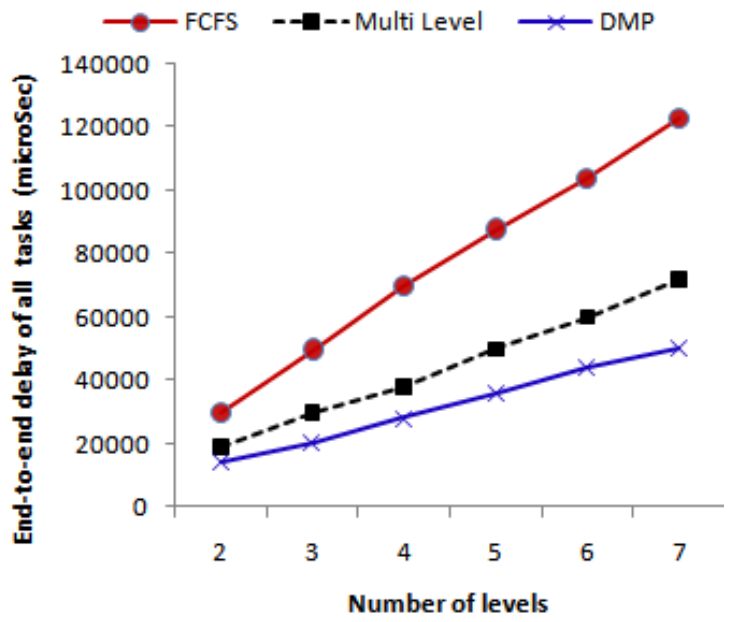

Fig.5 End to end delay of all type of data over no. of levels.

Fig. 5 demonstrate the end-to-end delay of all types of data traffic over a number of levels.

From these results, we find that the DMP task scheduling scheme outperforms FCFS, and Multilevel Queue scheduler in terms of end-to-end data transmission delay. This is because in the proposed scheme, the tasks that arrive from the lower level nodes are given higher priority than the tasks at the current node. Thus, the average data transmission delay is shortened.

\section{Conclusion \& Future Work}

In this paper, we propose a Dynamic Multilevel Priority (DMP) packet scheduling scheme for Wireless Sensor Networks (WSNs). The scheme uses three-level of priority queues to schedule data packets based on their types and priorities. It ensures minimum end-to-end data transmission for the highest priority data while exhibiting acceptable fairness towards lowest-priority data. Experimental results show that the proposed DMP packet scheduling scheme has better performance than the existing FCFS and Multilevel Queue Scheduler in terms of the average task waiting time and end-to-end delay.

As enhancements to the proposed DMP scheme, we envision assigning task priority based on task deadline instead of the shortest task processing time. To reduce processing overhead and save bandwidth, we could also consider removing tasks with expired deadlines from the medium. Furthermore, if a real-time task holds the resources for a longer period of time, other tasks need to wait for an undefined period time, causing the occurrence of a deadlock. This deadlock situation degrades the performance of task scheduling schemes in terms of end-to- end delay. Hence, we would deal with the circular wait and preemptive conditions to prevent deadlock from occurring. We would also validate the simulation result using a real test-bed. 


\section{Journal Papers:}

\section{References}

[1]. Nidal Nasser, Lutful Karim \& Tarik Talib, "Dynamic Multilevel Priority Packet Scheduling Scheme for wireless sensor network", IEEE Trans on wireless communication, vol 12, NO. 4, April 2013

[2]. G. Anastasi, M. Conti, and M. Di Francesco, "Extending the lifetime of wireless sensor networks through adaptive sleep," IEEE Trans. Industrial Informatics, vol. 5, no. 3, pp. 351-365, 2009.

[3]. G. Bergmann, M. Molnar, L. Gonczy, and B. Cousin, "Optimal period length for the CQS sensor network scheduling algorithm," in Proc. 2010 International Conf. Netw. Services, pp. 192-199.

[4]. E. Bulut and I. Korpeoglu, "DSSP: a dynamic sleep scheduling protocol for prolonging the lifetime of wireless sensor networks," in Proc. 2007 International Conf. Advanced Inf. Networking Appl., vol. 2, pp. 725-730.

[5]. S. Chachra and M. Marefat, "Distributed algorithms for sleep scheduling in wireless sensor networks," in Proc. 2006 IEEE International Conf. Robot. Autom., pp. 3101-3107.

[6]. P. Guo, T. Jiang, Q. Zhang, and K. Zhang, "Sleep scheduling for critical event monitoring in wireless sensor networks," IEEE Trans. Parallel Distrib. Syst., vol. 23, no. 2, pp. 345-352, Feb. 2012.

[7]. F. Liu, C. Tsui, and Y. J. Zhang, "Joint routing and sleep scheduling for lifetime maximization of wireless sensor networks," IEEE Trans.Wireless Commun., vol. 9, no. 7, pp. 2258-2267, July 2010.

[8]. J. Liu, N. Gu, and S. He, "An energy-aware coverage based node scheduling scheme for wireless sensor networks," in Proc. 2008 International Conf. Young Comput. Scientists, pp. 462-468.

[9]. O. Khader, A. Willig, and A. Wolisz, "Distributed wakeup scheduling scheme for supporting periodic traffic in wsns," in Proc. 2009 European Wireless Conf., pp. 287-292.

[10]. B. Nazir and H. Hasbullah, "Dynamic sleep scheduling for minimizing delay in wireless sensor network," in Proc. 2011 Saudi International Electron., Communications Photon. Conf., pp. 1-5.

[11]. D. Shuman and M. Liu, "Optimal sleep scheduling for a wireless sensor network node," in Proc. 2006 Asilomar Conf. Signals, Syst. Comput., pp. 1337-1341.

[12]. S. Paul, S. Nandi, and I. Singh, "A dynamic balanced-energy sleep scheduling scheme in heterogeneous wireless sensor network," in Proc.2008 IEEE International Conf. Netw., pp. 1-6, 2008.

[13]. A. R. Swain, R. C. Hansdah, and V. K. Chouhan, "An energy aware routing protocol with sleep scheduling for wireless sensor networks," in Proc. 2010 IEEE International Conf. Adv. Inf. Netw. Appl., pp. 933-940.

[14]. Y. H. Wang, Y. L. Wu, and K. F. Huang, “A power saving sleep scheduling based on transmission power control for wireless sensor networks," in Proc. 2011 International Conf. Ubi-Media Comput., pp. 19-24.

[15]. Y. Wang, D. Wang, W. Fu, and D. P. Agrawal, "Hops-based sleep scheduling algorithm for enhancing lifetime of wireless sensor networks," in Proc. 2006 IEEE International Conf. Mobile Adhoc Sensor Syst., pp. 709-714.

[16]. Y. Xiao, H. Chen, K. Wu, B. Sun, Y. Zhang, X. Sun, and C. Liu, "Coverage and detection of a randomized scheduling algorithm in wireless sensor networks,” IEEE Trans. Comput., vol. 59, no. 4, pp. 507-521, Apr. 2010.

[17]. X. Xu, Y. H. Hu, J. Bi, and W. Liu, "Adaptive nodes scheduling approach for clustered sensor networks," in Proc. 2009 IEEE Symp. Comput.Commun., pp. 34-39.

[18]. Y. Zhao, J. Wu, F. Li, and S. Lu, "VBS: maximum lifetime sleep scheduling for wireless sensor networks using virtual backbones," in Proc. 2010 IEEE INFOCOM, pp. 1-5.

[19]. B. Zeng, Y. Dong, and D. Lu, "Cooperation-based scheduling algorithm in wireless multimedia sensor networks," in Proc. 2011 International Conf. Wireless Commun., Netw. Mobile Comput., pp. 1-4.

[20]. N. Edalat, W. Xiao, C. Tham, E. Keikha, and L. Ong, "A price-based adaptive task allocation for wireless sensor network," in Proc. 2009 IEEE International Conf. Mobile Adhoc Sensor Syst., pp. 888-893.

[21]. H. Momeni, M. Sharifi, and S. Sedighian, "A new approach to task allocation in wireless sensor actor networks," in Proc. 2009 International Conf. Computational Intelligence, Commun. Syst. Netw., pp. 73-78.

[22]. F. Tirkawi and S. Fischer, "Adaptive tasks balancing in wireless sensor networks," in Proc. 2008 International Conf. Inf. Commun. Technol.: From Theory Appl., pp. 1-6.

[23]. X. Yu, X. Xiaosong, and W. Wenyong, "Priority-based low-power task scheduling for wireless sensor network," in Proc. 2009 International Symp. Autonomous Decentralized Syst., pp. 1-5. 